\title{
XXXII. Chemical inquiries into the nature of several fatty substances, and particularly on their combinations with the alkalis
}

\section{Chevreul}

To cite this article: M. Chevreul (1814) XXXII. Chemical inquiries into the nature of several fatty substances, and particularly on their combinations with the alkalis, Philosophical Magazine Series 1, 44:197, 193-206, DOI: 10.1080/14786441408637439

To link to this article: http://dx.doi.org/10.1080/14786441408637439

冓 Published online: 27 Jul 2009.

Submit your article to this journal $₫$

Џll Article views: 2

Q View related articles $\sqsubset$ 
and interior lining. In consequence I felt for some time after an unpleasant burning sensation in my throat, but neither purgative nor emetic effect followed. I afterwards repeated the six-grains lose, having previously removed the germ and paperlike lining, and with much diffeuity obtained one stool little differing from the natural.

I have not at present prosecuted these researches further: but, should cireumstances admit of $n=y$ following up these prelininary experiments, I slall lose no time in communicating their results to you for the gencral information of the medical world. The mut of the Sand Box is extremely palatable, tasting much like a good filbert.

An American captain who was in the habit of visiting Saint Kitts, before the war, was in the constant practice of using one or more of the seeds of the common Physic Nut (Jatropha Curcas) as a purgative when his bowels were irregular, using only the precaution of separating the germ, in which the violently drastic effects seemed solely to reside.

I have the honour to remain, With ligh esteem, sir,

Your most obedient servant, William Hamilton, M.B.

XXXIl. Chemical Inquiries into the Nature of several fatty Sulstances, and particularly on their Combinations with the Alkalis. By M. Cuevrrút*.

Of a new Sulstance, called Margarine, obtained from the Soats made from the Fat of Pork and Potash.

1. $\Gamma_{H E}$ combination of a fat substance with an alkali, presenting several products which are of great utility in the arts and domestic economy, was examined with respect to their usual properties: but in point of theory they were not made the subject of any special inquiry, so that we are reduced to hypotheses in order to explain one of the most common operations in our manufactories. The chemists of Stahl's school, who thought that the acid obtained from distilled oils was one of their constituent principles, generally regarded it as the cause of saponification. This idea was renounced when Lavoisier demonstrated that most of the bodies produced from organic matter, when distilled, resulted from a decomposition produced by caloric. Previous to Lavoisier M. Berthollet had regarded saponification in a more simple and accurate manner, by making it depend on

* Annales de Chimie, tome Ixxxviii. p. 225.

Yol. 44. No. 19\%. Sent. 1814. 
the affinity of the oil itself for the alkalis : so that soaps must be considered as compounds in which salifiable bases were nentralized by fat substances, the action of which was andogous to that of the acids : it was in this respect that he compared the oils to the latier substances, and we shall presently see how far this comparison was just. This way of thinking was adopted: but afterwards they went further, and pretended that the cil was not saponified but by absorbing oxygen: they did not found their opinion upon any positive fact, the slightest indications appearing sufficient.

2. In the present state of chemistry, however, we must not content ourselves with bare assertions: to establish a theory, we must be acquainted with the bodies which are in contact, analyse the compounds which they have formed, and see if the principles separated from it are the same with those which have been put in reaction. In commencing the account of my experiments on this substance, I find the double advantage of fixing the attention upon one of the most common products of the saponification of animal fat, and at the same time facilitating the study of fatty substances, by making known a species which possesses their generic properties in the highest possible degree.

3 . When we immerse soap made of pork grease and potash in a large quantity of water, one part is dissolved, while another is precipitated in the form of several brilliant pellets, which I shall call mother of pearl substance.

\section{Of the Purification of the Mothor of Pearl Sulstance, and of its Decomposition ly the Muriatic Acid.}

4. Eighty grammes $3 \frac{1}{4}$ oz. or 1551 grains English) dried in the air were diluted in 12 litres ( 3 gallons $1 \frac{5}{100}$ pints English) of water, and exposed to a temperature ranging from $50^{\circ}$ to $60^{\circ}$ : they absorbed this liquid, and increased greatly in size. At the end of ten days, the matter was put upon a filter; when it was drained, twenty litres of cold water was thrown over it, to deprive it of any soluble soap which it might retain. When thus washed it was dried, then treated thrice successively with two litres of boiling alcohol of the gravity of 0.820 divided into three equal portions : the first congealed almost wholly upon cooling; the second was slightly disturbed, and the third aimost not at all. The residue insoluble in alcohol weighed rather more than two grammes (about $38 \frac{1}{2}$ grains) ; it resembled the mother of pearl suhstance: it was different, however, from the part which was dissolved. The experiment tanght me that the mother of pearl substance was formed of a fatty substance, absolutely new, combined with potash, lime, and oxide of iron; and that when we 
treated it with boiling alcohol * we dissolved the conbination of potash, and a litice of those of lime and cxide of iror, while the latter, insoluble or much less soluble than the former, formed the residue.

I convinced mys If of these facts by treating in the following way the depositions which were separated from the alcoholie washings by cooling and concentration $\uparrow$ conpuratively with the residue. I put some very dilnte muriatic aci into two porcelain capsu.es : to the one I addad some mother of pearl su'stance which was soluble in alcohol, and to tite other the residue. On heating them the acid was combines with the salifiable bases, and the melted fatty su'sstance was separated from the liquid. After kceping this substance in fusion in dictilled water, and leaving it to become solid by cooling, I added the washings to the acid liquirls, and evaporated to dryness. I foun! that 100 parts of soluble matter had given to the muriatic acid 0.06 of lime and oxide of iron, and 5.07 of potash, while the residue had given to this acid lime, oxide of iron, and a mere atom of potash.

5. The fatty substance, separated from the salifiable bases, was dissolved in boiling alcohol: on cooling, it was obtained crystallized and very pure, and in this state it was examined. $\Lambda s$ it has not been hitherto described, it ought to be distinguished from other substances by a peculiar name: consequently I purpose to call it margarine, from the Greek word signifying pearl, because one of its characters is to have the appearance of mother of pearl, which it communicates to several of the combinations which it forms with the salifiable bases.

\section{Of Margarine.}

6. It is of a pearly white, and tasteless. Its smell is feehle, and a little similar to that of white wax. It is lighter than water. At $45 \cdot 2 \cdot 5$ Reaumur it melts into a very limpid colourless liquirl, which crystallizes upon cooling into brilliant needles of the finest white.

7. When we distilled it, it melted, and exhaled a white vapour which was deposited in a farinaceous-like matter in the neck of the retort. It boiled, and soon afterwards emitted an invisible vapour,

* A process smmewhat similar bas been adopted in this country to make what is called "transparent soap."-Travslator.

+ Every washing was separated from its deposition, and the filtered liquor was concentratcd to two-thirds its primitive volune, afterwards cooled and filtered. The rlepositations in the filter were washed with cold alcohol, then pressed in joseph paper, and by this means I obtained the rother of pearl substance in a state of purity. 
which was condensed into a liquid, and then into a white concrete mass. At the same time there was produced a little acid water, which was indebted for this property to vinegar, and perhaps some sebacic acid, and a strong smell, which I am inclined to attribute to a combination of volatile empyreumatic oil and acetic acid. When the matter contained in the retort began to turn black, and the product extricated from it was coloured yellow, I changed the recipient, and continued the fire until there was no longer any thing volatilized; very little gas or liquid was formed, and almost the whole of the product was solid. Four grammes (61 $\frac{3}{4}$ grains) of margarine yielded $0.045 \mathrm{gr}$. (about .3432 grain) of charcoal, containing an atom of lime, iron and potash, a first product, which was very white, weighing $2.19 \mathrm{gr}$. (33 grains), a second product, coloured yellow, weighing $1 \cdot 45 \mathrm{gr}$. (22 grains).

8. 'The first product boiled with half its weight of potash dissolved in water combined with the alkali. The combination diluted in water deposited a good deal of pearly matter. Hence it follows that this product was formed in a great measure of non-decomposed margarine.

9. The second product treated in the same way gave ont an atom of ammonia, which I thought accidental : instead of being combined with potash, it melted into a yellow oily liquid, which was again boiled with double its weight of alkali, and which refused to unite with it. After these two operations it weighed one gramme, and there was of course a loss : it had given little or nothing to the potash. When it was heated in alcohol it liquefied: a great quantity of this liquid is necessary to dissolve it: upon cooling, small crystals were precipitated of a pearly citrine white, which became of a slight yellow by fusion : the alcohol from which the latter were precipitated, when evaporated, left an oil of a deep yellow, which was liquid at $18^{\circ}$ centigrade. If the second product contained margarine, it must be coneluded that it was there in a particular state of combination, since the concrete crystallized substance and the liquid substance differ from it in every respect.

10. The alkaline liquors which had been boiled with the two foregoing products were united, concentrated, and filtered several times. They contained very little fatty matter in solution: when distilled with sulphuric acid, they gave an atom of vinegar, and a resilue containing some small crystals which had the appearance of sebacic acid.

11. Margarine is insoluble in water.

12. It is extremely soluble in alcohol; for 100 parts of the latter of the gravity of 0.516 dissolved at $75,180.79$ parts. 
This solution was only disturbed at $41^{\circ}$ of the centigrade. Upon cooling it congealed into a solid mass: this mass had a greenish shade, which became particularly cvident in those parts of the centre where a hole was made with a glass spatula. This phænomenon seemed to be owing to the alcobol, for the colour disappeared on the evaporation of this liquid. Whon the margarine is deposited by a gradual cooling, from a solution which is not saturated with it, it crystallizes in small neefles which unite in stars.

\section{Action of Potash on Margarine.}

13. I now come to speak of one of the most remarkable combinations of margarine, being that which it forms with potash. It truly characterizes this subitance, and leads me to examine some points of the ehemisal doctrines respeoting acidity.

14. When explaining as above the process for purifying the mother of pearl substance, I have observed that the latter gave me $8.07 \mathrm{gr}$. per cent. of potash; but having found alkali in the charcoal of distilled margarine, and being thereby convinced that the muriatic acid had not completely decomposed the mother of pearl substance, I wished to make a new analysis of it. I took two grammes of this substance perfectly pure and dried. I decomposed them by the muriatic acid, and kept the margarine several times in fusion in acidulated water, in order to take from it all its alkali. I obtained $0.255 \mathrm{cr}$. of muriate of dry potash, which represented 0.1032 of potash, almitting 64 of base in the muriate of potash. The dried margarine weighed $1 \cdot 865 \mathrm{gr}$. after having been fused. The 0.0282 exceeding the weight of the matter analysed, ought to be attributed to water remaining in the margarine, if the calculation of the muriate of potash which we have admitted be exact. In order to assure myself that the muriatic acid had dissolved all the potash united to the margarine, I charred two grammes of mother of pearl matter in a small platina crucible; I lixiviated the charcoal, and afterwards incinerated it: the atom of ashes which it left was mixed with the lixivium of charcoal. I combined the whole with the muriatic acid, and obtained 0.253 of muriate of potash, which confirms the first result. I think from this that we maystate the following as the composition of the mother of pearl suljstance :



15. The pearly matter is soft to the touch, and has no perceptible taste.

16. When heated in the sand bath, it does not melt; it bcN 3 gins 
gins to turn soft, however, and the lumps of matter get joined together.

\section{Action of Lrater.}

17. It does not undergo any action from cold water, for it preserves all its physical properties after the maceration of a month in this licquid: nevertheless, if we cxamine the latter, we find in it a small particle of alkali, and some almost imperceptible traces of pearly substance. Hot water has a more decided action on it: thus, when we put into 100 grammes of hoiling water one gramme of pearly substance, the latter becomes semitransparent, it resembles flakes of alumine recently precipitated from a very dilute solution. The water filtered when boiling does not pass over clear; if before filtering it we allow it to cool, it becomes turbid, and deposits mother of pearl substance: if we then filter it, we find very litule potash in it. 1.5 litre of water ( 3.1699 pints English) in which I had boiled 20 grammes of pearly matter, filtered after having been cooled and evaporated, lelt a residue of subcarbonate and of pearly matter which scarcely weighed $0 \cdot 1 \mathrm{gr}$, ( $\left.1 \frac{\mathrm{L}}{2} \mathrm{grain}\right)$. The matter deposited from the boiling water retains a little water, which gives it the property of melting at a temperature of $100^{\circ}$ centigrade, and a semi-transparency analogous to that of wax. I am strongly inclined to think that the pearly matter is not dissolved in hot water, and that it is only divisible in it by combining with water. I formed my opinion upon 1000 parts of water being boiled for two hours over one part of matter, without making it disappear.

18. The mother of pearl substance is less soluble in alcohol than marcarine. 100 grammes of this liquid of a gravity of 0.834 only dissolved 31.37 at a temperature of $67^{\circ}$ centigrade. This solution becomes turbid upon cooling; and this ought to be the case, since one part of mother of pearl matter requires 318 of alcohol to be dissolved at the temperature of $20^{\circ}$ centigrade: it does not chauge the colour of hematine; which proves that the potash is more strongly attracted by the margarine than by the colouring principle.

19. When we mix this solution with water, an abundant precipitate is formed: the sediment contains less potash than pearly matter : this is demonstrated by the following experiment. I dissolved in briling alcohol $4 \cdot 40 \mathrm{gr}$. of pearly matter containing $4.04 \mathrm{gr}$. of margarine and 0.36 potash. I poured the liquor while still warm into 1.5 litre of water, and shook the misture several times. In twelve hours I filtered: the liquid evaporated deposited an inappreciable quantity of pearly matter: 
it contained $0.052 \mathrm{gr}$. of potash : consequently, the margarine and the alkali of the matter precipitated muss have been there in the proportion of 100 to $7 \cdot 62$. The analysis which I made with the muratic acid gave me the proportion of 100 to 7.95 , which differs from the former only by 0.33 . If we take the mediun, we shall have $7 \cdot 78$, and we shall tlien find that one-eighth of the alkali contained in the pearly matter was scparated by the water.

20. The matter which was precipitated from the alcohol by the water did not yield a perceptible quantity of alkali to the latter; but if we add a little hematine, a part is then sepurated. 'This is proved by the two following experinents. I boiled the matter in wat $\wedge$, divided the liquid into two equal portions, filtered one of them, and afterwards put hematine into the filtered liquor, and also into that which was not filtered. The former makes almost no change on the colouring principle, whereas the latter renders it purple by giving alkali to it. This decomposition takes place only in consequence of the united action of the water and bematine; for, if we mix the dissolved matters in alcohol, the hematine undergoes no change. (18.)

21 . The matter which was precipitated from the alcohol by water, redissolved twice in the alcohol, was deposited upon cooling in the state of true mother of pearl matter, which contains 100 of margarine and 8.88 of potash.

22. It results from what I have said on the presipitation by means of water from the alcoholic solution of mother of pearl substance: 1st, that in this precipitation there is a cuantity of potash separated from the nargarine, which seems to be oneeighth of that which is combined with it ; that this separation is owing to the alfinity of water for alcohol and the potash, and to the insolubility of the margarine in water. If boiling water takes less alkali from the mother of pearl substance than cold water poured into the alcoholic solution of the latter, this seems to arise, because in the second case this liquid exercises its action on a dissolved body; whereas in the other it exercises it on a colirl, the cohesion of which is an obstacle to its solvent power. $2 d$, Thit the matter presipitated from alcohol yields little if any alkali to boiling water, but yields it to an aqueous solution of hematine. 3dly, That by redissolving it in boiling alcohol, it is precipitated in mother of pearl matter unon cooling. This proven, that in this substance the two elements exist in a proportion in which they enjoy a conesion sufticient to determine the separation of the excess of margarine fron which the water had taken the alkali.

23. This last consirieration led me to inquire if margarine, when presented to a hot solution of potash holdung a quantity of alkali much more consicterahle than that which was necessary 
to convert it into pearly matter, should be changed into this matter, or rather into a more aikaline combination.

24. I put into 160 grammes of water holding 24 grammes of alcoholized potash, 40 grammes of ma:sarive. The latter became soft by heat, sthatinous, and semi-tranparent, by uniting with the potash. The substances put in digastion for six hours, at the temperature of $80^{\circ}$ or $90^{\circ}$ centiarade, were aifterwards allowed to stand by theneelves. In fifteen hours a white and opaque mass was separated from an almost colourless mother water. This licuid supersaturated with sulphuric neid deposited a small particle only of marrarine, and yielded upon distillation neither acetic acid nor volatile oil.

25. The white was separated from the mother water was pressed betricen yices of joseph paper, until no more liquid was yielded : it was then white and opaque. This method appearing to me to be insufficient for taking up all the alkali which was not in combination with the margarine, 1 found, after several fruitless experiments, that boiling alcohol dissolved the substance very well, and that upon cooling it was deposited in the form of small needles, which it was very easy to obtain in a state of perfect purity by putting them on : i filter, washing them with cold alcohol, then squeezing them in joseph paper, and exposing them for several hours to the rays of the sun.

26 . Two grammes of these needles, thus treated, decomposed by the muriatic acid, gave $1.72 \mathrm{gr}$. of margarine, and $0.475 \mathrm{gr}$. of muriate of potash, representing 0.3072 of base. If we admit that the excess of weight is owing to water retained by the margarine, we shall have the following proportion:

$$
\begin{aligned}
& \text { Margarine ......... 100 } \\
& \text { Potash ........ 15.14 }
\end{aligned}
$$

This result shows that when we unite directly the margarine with potash, there is a combination formed which contains twice as much alkali as pearly matter; for we have found this last formed of 100 parts margarine, and 8.88 of potash. Now this quantity multiplied by two, gives $17: 76$ which differs only in 0.38 from the foregoing determination. Margarine therefore in these combinations with potash obeys the same laws with the inorganic bodies.

27. The combination of margarine saturated with potash presents the following properties:

28. It is white, and not so soft to the touch as the pearly matter. It has a very slight alkaline taste.

29. When we put it into pure water, it is decomposed into a pearly matter and into potash. We may be convinced of this by putting it into abundance of cold water, and shaking the mixture from time to inie. The matter thrown upon a filter and washed yiclded 


\section{fatly Substances, and on their Combinationswith the Alkalis. 201}

vielded margarine 100 , and potash 8.55 . The washings when filtered and evaperated contained potash and a trace of margarine. If instead of putting the combination of margarine saturated with alkali in plenty ot water, we fut it in a small quantity of this liquid, it swells, becomes semi-transparent by absorbing ic, and forms a thick mucilage which exhibits pearly matter when it is shaken. In this ease the mass of water not being sufficient to overcome the whole si the afnity of the pearly matter for an excess of potash, it happens that the clecomposition of the saturated combination is only partial, and it is the part which is not altered which absoris the water, and forms with it a mucilaginous liquid, by retaining it among its molecules. If we throw the whole upon a filter, after having mixed enough of water with it to facilitate the filtration, annlysis demonstrates less potach than in the saturated combination, and more than in the pearly matter; which proves that the decomposition has been but partial.

30 . When we put three grammes of saturated combination in 100 grammes of boiling water, we obtiin a solution which is perfectly limpid while the liquer is warm, and which even may be filtered. This solution upon cooling deposits abundance of mother of pearl matter, and afterwards becomes thick. When it is completely cooled, it is precisely like cold water in which we have put a great quantity of pearly combination: only the mucilage is much more homogenecus. The filtered liquor contains potash and some atoms of margarime; for it is not reudered turbid by the acida, except when it has been concentrated.

31. It follows from what has been said: 1 . That the saturated combination of margarine is decomposed into mother of pearl substance, and into potash, by a great mass of cold water; $2 \mathrm{dly}$, that a little of this liquid only decomposes a part of it; that what is not decomposed absorbs water, and forms a thick semi-transparent mueilage; $3 \mathrm{dly}$, that when the action of the water is assisted by that of caioric, the saturated combination may be completely dissolved, and that by cooling there is formed pearly matter, and a thick mucilage of saturated combination, if the water he not always in excess.

32. The saturated combination is dissolved in boiling alcohol, and partly precipitated upon cooling, without undergoing decomposition. 100 parts of boiling alcohol of a gravity of 0.834 appeared to me to dissolve only 8.93. When we dilute this solution in water, we obtain pearly matter in the form of very brilliant small crystals. If the alcohol does not recompose the saturated combination as water does, this is owing to its dissolving equally well potash and margarine; whereas water dissolving only 


\section{Chemical Inquiries into the Nature of several}

only one of the elements of the combination, attracts a portion of the larter with more force than the insoluble element tigts.

33. Margarine decompones the subearbonate of potash. We may render the decomposition evirient, by passing into is tuise full of mercury a mixture of eight parts of water, one of margarme, and half a part of subcarbonate, and by heating it atiprwarks with a red-hot iron to ebullition. After cooling we lind a gaseous residue which is pure carbonic acid. By repating the experiment in a small phial furnished with a curved tube, I observed that the nargarine was dissolved before there was any extrication of carbonic gan, and that this extrication only took place when the liquor boiled. This circumstance induced me to think that, at the temperature at which margarine may be united with potash, the carbonic acid which is separated from it mar act upon a portion of subcarbonate, and convert it into saturated carbonate, and that it is afterwarts the latter which seinds out carbonic acid at the temperature of boiling water. The combination of margarine formed in this oyeration gave me, after having been washed, margarine 100 , potash 6.88 ; it was therefore pearly matter. The lingor from which it was separated, filtcred several times, presented only some particles of margarine, although it contained a great excess of alkaline carbonate.

Action of Margarine upm Turnsole.

34. The strong afinity of margarine for potash having macle me think that this substance condl redden tuinsole, I put three grammes into the aqueous extract of turnsole: when cold there was no action; but when heated the margarime becane soft, without melting however, and the blue colour became red. I decanted the cooled licpur, and boiled several times the solid matter which was separated from it with new extract of turnsole. I filtered, and there remained upon the paper red clots and a blue semi-gelatinous marter, which became partly red when dried: each of these substances was dissolved by boiling alcohol: the two solutions were red, and deposited upon coolings small erystals: those coming from the former yiekded margarine 100 , potash $7 \cdot 5$; those of the latter, margarine 100 , and potash 8.45. As I made these determinations on very small quantities only, I cannot vouch for their perfect accuracy; they are sufficient at least to establish that margarine takes up the potash from the colouring principle of turnsole, and that it acts like the acirls.

3.. The affinity of margarine for potash is not only sufficiently large to determine the formation of the pearly matter at the expense of the alkali of the turnsole dissolved in watcr, but it is also 
also sufficient for the pearly matter itself dissolved in alcohol seizing upon its alkali, and being converted into the combination of margarine saturated with potash. If we only obtain pearly matter instead of this last combination, by boiling margarine in t'le açueous extract of turnsole, this ought not to be surprising, if we recollect that the saturated combination is decomposed by water, and consequently it camot be formed in the midst of a great mass of this lifuid. This besides may be easily demonstrated by pouring water into turnsole which has been reddened by the alcolnolic solution of pearly matter : at the moment of the niisture, the colour passes to blue, because the water determines the pearly matter to give up to the colouring principle of the turnsole the alkali which it had at first taken from it.

36 . We have shown that margarine possesses a part of the characters of the acids; for it neutralizes the alkalinity, and attracts the potash with more force than the colouring principies do when employed as reagents. Thut are these properties sufficient to entitle us to place it anomg the acids? If we had fixed the properties which are essential to those bodies, it would be easy to decile this question; but as this has not been done, it is indispensable, before seeking to resolve it, to examine the most general characters wh ich have been ascribed to the acids.

37. These characters are six in number; viz. 1. A sour taste. 2. Deing capable of being attracted positively by electrified surfaces. 3. To neutralize more or less the salifiable bases. 4. To redden turnsole. 5. To rerkien the colour of violets. 6 . To tum yellow or redden hematine.

l. The sour taste was the first property which served to distinguish the acids: and this character is good; for it belongs to the greater number of those bodies; and, so far as I know, none of the substances which are regarded by chemists as non-acids possess it.

2 . If the second character seems common to all the acids, it is not exclusive to them; oxygen possesses it par excellence; and $M$. Berzclius asserts that sulphur, carbon, and arsenic act equally towards the suifaces positively electrified,

3 . While the sour taste was the first property assigned to the acids, at a period when chemistry did not yet esist, so that of nentralizing more or less the allsalis was one of the first which was regarded as the chief property, when chemical phænomena began to be understond. It was even supposed that there must be an acid principle in several bodies which united with the alkalis, and which in other respects differed extremely from the acids.

4. The property of reddeniug turnsole has been strongly insisted 
sisted upon; but this character does not differ essentially from the foregoing ; for, the turnsole being a combination of red colouring matter and potash, it happens that it is reddened by all the bodies whose affinity for alkali is superior to that of the cobouring matter. Consequently this reagent announces that the body which reddens it is susceptible of being united to potash with a greater force than its colouring matter; it does not therefore indicate acidity by itseif, it simply estallishes a relation of attraction between bodies which have afinity for the alkalis. The case is not the same with the indications by the violet colour of the hematine: those reagents announce the acidity by a change of colour resulting from the combination of the acid with the colouring principle itself.

5. Hitherto, the acids only have been sipposed to redden the colour of violets, but there are some which act differently : thus the boric acid and prussic acids do not redden it. In all probability, it is the sane with sulphuretted hydrogen.

6. The action of the acids on hematine is much more general than that which they exercise on the colour of violets: thus all of them make it pass to the yellow or the red, and there is scarcely one but sulphuretted hydrogen, which seems to make an exception; it forms with it a combination of a yellow so slight that it seems to be colourless when it is in a thin layer; and it ought to be adrled, that almost all the oxides which neutralize the acids act with it like the alkalis, with the excrption, however, of the oxide of tin at the maximum, which acts in the manner of an acid.

33. If we now compare the whole of these characters, we find:

1. That the sour taste does not belong to all the acids : consequently it has not been regarded as being essential to those bodies.

2. That the property of being attracted by surfaces positively clectriffed is too general to characterize acidity.

3. That the case is the same with the neutralization of the alkalinity; tor those chemists who have been most forcibly struck with this character have never formally said that it is sufficient for assigning acidity to a body which possesses it. If some of those chemists bave regarded sulphur as an acid, no person has ever ascerted that the oxides of lead and of zinc were in the same prerlicament: nevertheless, in ranking sulphur among the acids, there was no reason for separating from it the latter, which belong very eridently to the class of salifiable bases, since they form salts with the acids, and they react on hematine in the minner of potash, barytes, \&c.: hence it follows that the faculty of neutralizing an alkali does not convey the idea of acidity:

4. That 


\section{falty Sulstances, and on their Comlinations with the Alkalis. 205}

4. That although the indication of turnsole has not been appreciated at its true value, it is nevertheless this character which has been most generally employed, and that which chemists seem unanimously to have adopted by tacit consent; for none of the bodies reputed to be acid are deprived of it; and this character, which differs from the foregoing only in so far as it values the strength at a given point, has been sufficient to place several bodies among the acids, and to exclude others from the latter, because they did not possess it as at first supposed. I know but two oljections which can be made to this. The first is, that there may exist bodies which do not rchlen turnsole on actount of their cohesion, and which may nevertheless approach closely to the acids : the second is, that if we have clemonstrated that the property of neutralizing the alkalinity does not belong solely to the acids, we may suppose one of those bodies which, not having any other property of acidity, will redden turnsole, because it will attract its alkali more strongly than it does the colouring principle.

5. That the proverty of reddening the tincture of violets is not sufficiently common to entitle us to draw any conciusion relative to the bodies which do not possess it.

6. That although the indication of the hematine is much more general than the foregoing, it is nevertheless difficult to admit it exclusively, because there are acids almost insoluble, whose affinity for it is so weak that it is difficult to approach its action.

39. To resume our conclusions: We see in the first place, that turnsole appears to be adopted by all chemists to detect acidity. That if we do not pay some attention to the objections alluded to above as to its value, and if we persist in following the principles which have hitherto been taken as guides in the classification of the acids, we shall be compelled to rank margarine among the latter; since it reddens turnsole, takes up potish from the carbonic acid, and since its combinations with this base have the greatest analogy with the salts. If it be objected that its composition removes it too far from the series of the acids, we may cite a single example, that of sulphurettex hydrogen, which evidently possesses the characters of acidity, as $M$. Berthollet has proved. All the chemists, in regarding this body as an acid, have, I believe, established that in the chemical system the analogy of properties has been consulted in preference to that of composition. Secondly, that if we refuse to rank margarine among the acids, we are led to these conclusions: 1. That the taste and action of the colouring principles which undergo changes of colour by their immediate combination with the acids, 


\section{Chemical Inquiries into the Nature of fatty Substances.}

acids, arc the only characters admissible fot ascertaining acidity: that, in this case, we shall be forced to exclude one or other of these characters, since the oxide of tin at the maximum, without having an acid taste, acts on the hematine in the manner of an acid. Finally, that by determining for the colouring principles, we shall be reduced to the employment of hematilie only, since the tincture of violets has an action ton limited. 2. That the indication of turnsole being absolutely insignificant, we must submit to a new examination all the bodies which have been ranked among the acids, from the sole consideration that they were combined with the bases with sufficient force to reriden turnsole, that it is not doubtful that several of these bodies $n$ ill be separated from them. 3. That margarine ought to be ranked anong the fatty substances, without having any regard to its properties.

40. After having appreciated the indication of each of the characters of acidity in particular; after having demonstrated that some were too general, while others were too limited; I have detailed the corisequences to which we are led, according as we admit such or such of those characters. I have not deeided, because I have regarded my individual opinion as of little importance. Whatever may be the case, however, it is very remarkable to see a fatty substance in which hydrogen and carbon prevail, possessing in as marked a degree as certain acids, one of the noost characteristic properties of the latter. But let it not be supposed that margarine alone is in this predicament: I have already met with several analogous substances, which I shall describe in future papers : and the observation of Messrs. Bouillon Lagrange and Vogel, that the resins redden turnsole, proves that it exists completely formed in vegetables. These facts, therefore, permit me to think that we shall be able to make a group of fatty substances, the affinities of which for the alkaline bases will be analogous to those of the oxygenated acids, and whose combinations will present species of saline compounds to which we may give the generic name of soaps, which several of them have so long gone under. This analogy of properties which we observe between oxygenated and inflammable bodies is well adapted for supporting a principle which had already guided me in my experiments on astringent substances; namely, that an analogy of properties is not always a consequence of an analogy of composition. 